\title{
Ovarian remnant causing hemoperitoneum: a rare presentation ovarian remnant syndrome with review of literature
}

\author{
Analise D Mello, Rupa Padwalkar \\ Corresponding author: Dr. Analise D Mello , House number 14/4, Hillview estate, Gounlloy, \\ Nuvem , Salcete, Goa 403601, India; Email : analise2288@gmail.com
}

Distributed under Attribution-Non Commercial - Share Alike 4.0 International (CC BY-NC-SA 4.0)

\begin{abstract}
$\underline{\text { ABSTRACT }}$
Ovarian remnant syndrome (ORS) results from the presence of residual ovarian tissue after oophorectomy. The most common preexisting conditions include endometriosis, pelvic inflammatory disease and prior abdominal surgery as in these conditions the removal of ovarian tissue becomes difficult. It should be always considered as a differential diagnosis for chronic pelvic pain. A 50 year old postmenopausal multigravida female was diagnosed with hemoperitoneum. She had undergone hysterectomy with bilateral salpingoophorectomy for endometriosis 5 years ago. Laparotomy performed and a 10X16 cm hematoma along with ovarian tissue was evacuated and sent for histopathology which confirmed presence of ovarian cystadenoma tissue with hemorrhage that is consistent with the diagnosis of ORS.
\end{abstract}

Keywords: Ovarian remnant syndrome, hemoperitoneum, endometriosis.

Ovarian Remnant Syndrome (ORS) is defined as locating histologically confirmed ovarian cortical tissue during explorative surgery in a female patient who had previously undergone bilateral salpingo-oophorectomy and now presented with pelvic mass or pain ${ }^{1}$. The syndrome first described by Shemwell and Weed in 1970 and the first case presented in literature in 1962 was by Kauffman ${ }^{2,3}$. The problem arises when the ovary is incompletely resected as it was densely adherent due to endometriosis, extensive adhesions, or pelvic inflammatory disease and the remnant of ovarian tissue may get vascularised under influence of hormones. Cystic degeneration is also noted in the remnant due to compromised blood supply. Ovulation may occur causing bleeding thereby increasing the size of the remnant often mistaken for a surgical cause of the pathology due to the history of oophorectomy.

It is a rare condition. The earliest published reports are in 1962 and sporadic reports of the same associated with complications of urinary retention and intestinal obstruction have been published. The true incidence remains unknown and case studies have become more common due to awareness among doctors and imaging techniques available 4,5 . We present a case of ovarian remnant syndrome which was opened by the surgeons as a hemoperitoneum with a surgical cause after blunt abdominal trauma.

\section{Case}

A 50 year old para2 was admitted under department of surgery with history of severe abdominal pain following a fall at home. Patient had history of right sided lower quadrant abdominal colicky type pain increasing in intensity

Received: $12^{\text {th }}$ May 2020. Peer review completed: $4^{\text {th }}$ July 2020, Accepted: $12^{\text {th }}$ July 2020.

Mello AD, Padwalkar R. Ovarian remnant causing hemoperitoneum: a rare presentation ovarian remnant syndrome with review of literature. The New Indian Journal of OBGYN. 2021; 7(2): 251-53. 
since the fall. Patient gave history of undergoing a total abdominal hysterectomy and bilateral salpingooophorectomy 20 years ago for endometriosis.

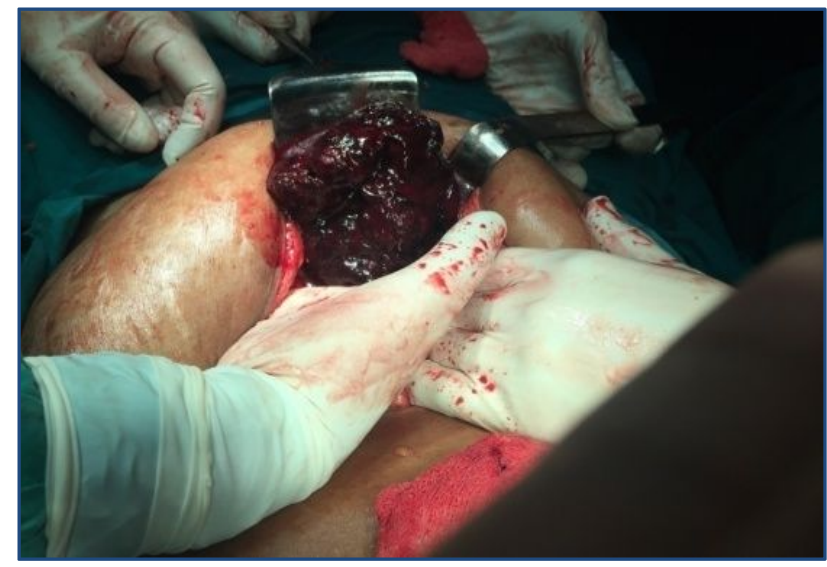

Figure 1: Clot removed from pelvis overlying ovarian tissue

On examination, patient had marked pallor, few coarse crepitations at the lung bases and on per abdominal examination horseshoe shaped dullness and tenderness on the right iliac fossa and presence of pfannenstiel scar was noted. On per vaginum examination a $7 \mathrm{~cm}$ mass felt on right side of vault whose upper extent could not be felt.

On ultrasonography and CECT scan, there was free fluid noted (hemoperitoneum) noted and a $20 \times 8 \mathrm{~cm}$ hematoma in the pelvis. The absence of uterus and adnexa confirmed the post operative status. In the view of the findings, decision was taken for a laparotomy.

On the operating table, 2 litres of hemoperitoneum was drained after dissecting dense adhesions of omentum to anterior abdominal wall and between bowel loops. A 500gm clot removed from pelvis overlying ovarian tissue (Figure 1) and right ovarian remnant excised after repairing a $3 \mathrm{~cm}$ rent in it. There was evidence of endometriotic deposits on vault and sigmoid colon and evidence of TAH and BSO. On histopathology examination of the tissue obtained, ovarian cystadenoma with hemorrhage was noted.

\section{Discussion}

The risk factors for ORS are endometriosis, pelvic inflammatory disease, prior abdominal surgery. Ovarian remnant undergo functional changes, increase in volume within fixed spaces of adhesions causing pain and compression of adjacent structures ${ }^{6-9}$. Within minimum 5 years of oophorectomy symptoms of abdominal pain, lower back pain, dyspareunia, variable bowel symptoms, pelvic masses, ureteral compression symptoms or incidental pelvic mass on routine checkup.

Investigations include CA 125 levels which are raised in malignant changes, FSH $<40 \mathrm{mIU} / \mathrm{ml}$ which is abnormal in perimenopausal range in patients who underwent BSO. Radiological investigations like ultrasonography suggestive of cystic pelvic mass with rim of vascularised tissue which will be confirmed on MRI. The common complications associated with ORS include malignant change in the ovarian remnant like endometroid adenocarcinoma and primary ovarian clear cell carcinoma ${ }^{10}$.

Oral contraceptive pills, GnRH analogues and MPA enable the ovarian remnant symptom suppression. Clomiphane citrate $100 \mathrm{mg}$ /day prior to surgery enables the visualization of the ovarian remnant by stimulating the ovarian tissue fragments and facilitating access and removal. Gabapentin used along with GnRH agonist for better pain relief and symptom suppression ${ }^{11,12}$. The other method is external beam radiation of 200cGy units in 10 fractions over 10 treatment days for a total of $2,000 \mathrm{cGY}$ is used in patients with ORS and there has been complete resolution in symptoms and the remnant ${ }^{13}$. Laparoscopy or robotic assisted better than laparotomy as the magnification allows better identification of anatomy, there is reduced blood loss and better visualization with increased intra abdominal pressure and it is less traumatic ${ }^{14}$.

Key steps to avoid ORS include restoring anatomy after completion of surgery, dissection of ureters and use of stenting preoperatively if required and lastly complete and thorough examination of abdominopelvic cavity performed before closure.

\section{Conclusion}

ORS is an uncommon complication of difficult oophorectomy. The preoperative diagnosis is challenging due to nonspecific clinical symptoms and imaging findings often mistaken for other non gynaecological causes. Therefore, ovarian remnant should be considered in differential diagnosis of a patient, presenting with chronic pelvic pain and pelvic mass with the history of difficult oophorectomy or suspected intraoperatively when the location and the cause do not correlate to any other surgical cause.

Acknowledgement: To the department of surgery, Goa Medical college, Bambolim, Goa

Conflict of interest: None. Disclaimer: Nil. 
The New Indian Journal of OBGYN. 2021 (January-June);7(2)

\section{References}

1. Magtibay P, Magrina J. Ovarian remnant syndrome. Clin Obstet Gynecol. 2006;49:526-34

2. Shemwell RE, Weed JC. Ovarian Remnant syndrome. Obstet Gynecol.1970; 36: 299-303

3. Kauffmann JJ. Unusual causes of extrinsic ureteral obstruction Part 1. J Urol. 1962; 97: 319-27

4. Nehzat C, Kaerney S, Malik S, Nehzat C, Nehzat F. Laproscopic management of ovarian remnant. Fertil steril. 2005: 83: 973-8.

5. Pettit PD, Lee RA. Ovarian remnant syndrome: diagnostic dilemma and surgical challlenge. Obstet Gynecol. 1988; 71: 580-3

6. Symmonds RE, Pettit P. Ovarian remnant syndrome. Obstet Gynecol. 1979; 54: 174-7

7. Lafferty HW, Angiolo R, Rudolph J, Penalver MA. Ovarian remnant syndrome: experience at Jackson Memorial hospital, University of Miami 1985-1993. Am J obstet gynecol. 1996; 174: 641-5

8. Webb MJ. Ovarian Remnant syndrome. Aust N ZJ Obstet Gynecol. 1989; 29:433-5

9. Steege JF. Ovarian remnant syndrome. Obstet Gynecol. 1987; 70: 64-7
10. Ichigo S, Takagi H, Matsunami $\mathrm{K}$, Murase $\mathrm{T}$, Ikeda $\mathrm{T}$, Imai A. Clear cell carcinoma developing in remnant ovary after hysterectomy and bilateral salpingooophorectomy for endometriosis. Journal of Gynecologic Surgery. 2014; 30(2): 114-7

11. Orford V, Kuhn R. Management of ovarian remnant syndrome. Aust N Z J Obstet Gynecol. 1996; 36: 468-71

12. Soares LC, de Souza RJ, Brollo JLA. Ovarian remnant syndrome: A case report. J Reprod med Gynecol Obstet. 2016; 1: 002

13. Zimmerman P. Unusual use of radiation therapy for pelvic pain. Clinical Advisor. June 2014

14. Kamprath S, Possover M, Schneider A. Description of laproscopic technique for treating patients with ovarian remnant syndrome. Fertil Steril. 1997; 68: 663-7
Analise D Mello ${ }^{1}$, Rupa Padwalkar ${ }^{2}$
${ }^{1}$ Assistant Professor, Department of Obstetrics and Gynaecology, Pondicherry Institute of Medical
Sciences, Kalapet, Puducherry, India; ${ }^{2}$ Lecturer, Department of Obstetrics and Gynaecology, Goa Medical College, Bambolim, Goa, India. 